\title{
Severe aortic regurgitation due to a Lunderquist Extra-Stiff Wire Guide during transcatheter aortic valve implantation
}

\author{
Daisuke Hachinohe $e^{1,2,3}$, Marco B. Ancona ${ }^{1}$, Antonio Colombo ${ }^{1,2}$, Azeem Latib ${ }^{1,2}$ \\ ${ }^{1}$ Interventional Cardiology Unit, San Raffaele Scientific Institute, Milan, Italy \\ ${ }^{2}$ Interventional Cardiology Unit, EMO-GVM Centro Cuore Columbus, Milan, Italy \\ ${ }^{3}$ Cardiology Department, Sapporo Heart Center, Sapporo Cardio Vascular Clinic, Sapporo, Japan
}

An 85-year-old female with severe aortic stenosis was referred for transfemoral transcatheter aortic valve implantation (TAVI). Initial aortography showed trivial aortic regurgitation (AR) (Fig. 1A, Suppl. Video 1). After insertion of a Lunderquist Extra-Stiff Wire Guide (LES; Cook Inc., USA) into left ventricle, blood pressure decreased gradually to $60 \mathrm{mmHg}$, unresponsive to catecholamines. There was no evidence of left ventricular perforation, exacerbation of mitral regurgitation, or peripheral artery perforation. Aortography revealed severe AR and immobile right coronary leaflet (Fig. 1B, Suppl. Video 2). After removal of LES and switching to a pigtail catheter, vitals and AR improved immediately (Fig. 1C, Suppl. Video 3). Finally, a $29 \mathrm{~mm}$ Portico (St. Jude Medical, Inc.,
USA) was implanted with Safari wire (Boston Scientific, USA) (Fig. 1D).

Guidewires with moderate stiffness compared to LES tend to go in line with a patient's anatomy and pass toward non-coronary cusp and settle within the commissure between right-coronary and non-coronary cusp. In this case, LES might have blocked the right coronary leaflet, leading to severe AR. LES, which has more than double the stiffness than other guidewires, tends to remain straight rather than along a patient's anatomy and may be more likely to go toward right-coronary cusp (Fig. 1E, F). When using an LES, efforts to exclude severe AR if patient vitals deteriorate is important in preventing disastrous complications.

Conflict of interest: Dr. Azeem Latib is a consultant for Medtronic. All other authors have reported that they have no relationships relevant to the contents of this paper to disclose.

Address for correspondence: Azeem Latib, MD, IRCCS San Raffaele Scientific Institute, Via Olgettina 60, 20132 Milan, Italy, tel: +390226437331, fax: +390226437339, e-mail: alatib@gmail.com

Received: 10.05.2018 Accepted: 18.06.2018 


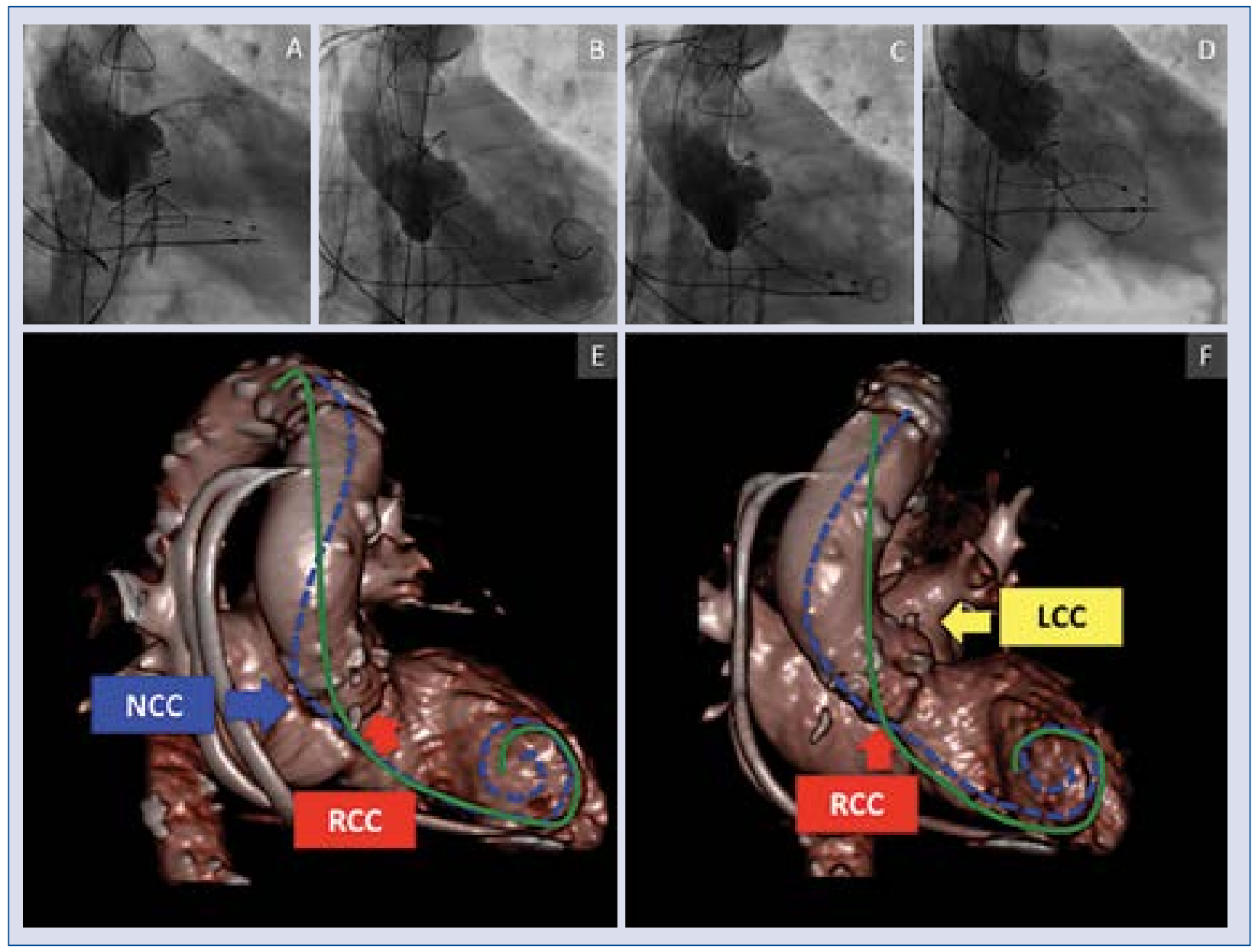

Figure 1. Aortography during transcatheter aortic valve implantation and computed tomography (CT) reconstruction (A) Initial aortography shows trivial aortic regurgitation (AR) (Suppl. Video 1). B. Aortography after insertion of a Lunderquist Extra-Stiff Wire Guide in left ventricle (LV) reveals severe AR and immobile right coronary leaflet (Suppl. Video 2). C. Immediately after removal of the Lunderquist wire from LV, aortography shows improvement of AR (Suppl. Video 3). D. Final aortography shows an excellent result. Three-dimensional CT reconstruction of right anterior oblique projection (E) and anterior-posterior projection (F). Green solid lines indicate the Lunderquist wire position, going straight rather than being along the patient's anatomy. Blue dotted lines demonstrate the expected position of the other guidewires, passing toward non-coronary cusp (NCC); LCC — left-coronary cusp; RCC — right-coronary cusp. 